\title{
Valoración médico legal de la fibromialgia en el campo de la patología laboral
}

Legal medical evaluation of fibromyalgia in the field of occupational disease

\section{María Ibáñez Bernáldez ${ }^{1}$, Mariano Casado Blanco², Raquel Miranda Gutiérrez ${ }^{3}$}

Resumo: Com a denominação de fibromialgia (FM) ou síndrome da fibromialgia (SFM) queremos significar aquela entidade clínica caracterizada por um quadro de dor músculo esquelético crónico e generalizado de origem desconhecida para o qual não existem outras doenças ou alterações que o expliquem. A própria definição da doença, seus critérios diagnósticos e possíveis fatores etiológicos são ainda objeto de debate no momento atual. Objetivos: Revisão da jurisprudência existente a respeito das situações geradoras de incapacidade em pacientes afetados por esta doença. Conclusões: A avaliação da incapacidade nos doentes com FM é geralmente um processo cheio de controvérsias, tais como a incredulidade diagnóstica de alguns dos agentes avaliadores, a participação de anomalias psicológicas, a dificuldade na objetivação da incapacidade, a deficiência dos instrumentos de avaliação e a baixa eficácia do tratamento.

Palavras-chave: Fibromialgia; Doença do trabalho; Valorização médico-legal.

\begin{abstract}
Under the name of fibromyalgia or fibromyalgia syndrome we mean that clinical entity characterized by a muscleskeletal chronic and widespread painful condition of unknown origin, for which there are no other diseases or disorders to explain it. The very definition of the disease, its diagnostic criteria and the possible etiologic factors are still debated at the present time. Objectives: Review the existing jurisprudence about the situations that generate disability in patients affected by this illness. Conclusions: The assessment of disability in patients with FM is usually a process fraught with controversy, for reasons such as diagnostic disbelief for some of the reviewers agents, the occurrence of psychological abnormalities, difficulty in objectifying disability the deficiency assessment tools and poor treatment efficacy.
\end{abstract}

Keywords: Fibromyalgia; Occupational Disease; Medical-legal Assessment.

Resumen: Con la denominación de fibromialgia (FM) o síndrome de la fibromialgia (SFM) queremos significar aquella entidad clínica caracterizada por un cuadro de dolor músculo esquelético crónico y generalizado de origen desconocido y para el que no existen otras enfermedades o alteraciones que lo expliquen. La propia definición de la enfermedad, sus criterios diagnósticos y posibles factores etiológicos son todavía objeto de debate en el momento actual. Revisión de la Jurisprudencia existente acerca de las situaciones generadoras de incapacidad en pacientes afectos por esta dolencia. Se desarrolla, a partir

\footnotetext{
1 Doctora en Medicina. Profesora de Medicina Legal. Facultad de Medicina. Universidad de Extremadura. Badajoz, España. E-mail: maria.ibanez@justicia.es

2 Doctor en Medicina. Profesor de Medicina Legal. Facultad de Medicina. Universidad de Extremadura. Badajoz, España.

${ }^{3}$ Fisioterapeuta. Facultad de Medicina. Universidad de Extremadura. Badajoz, España.
} 
del estudio de la repercusión que esta enfermedad crónica tiene en el campo de la patología laboral, los tiempos medios de incapacidad temporal y como causa de incapacidad permanente. La evaluación de la discapacidad en los enfermos con FM es generalmente un proceso plagado de controversias por razones tales como la incredulidad diagnóstica de algunos de los agentes evaluadores, la concurrencia de anomalías psicológicas, la dificultad en la objetivación de la discapacidad, la deficiencia de los instrumentos de evaluación y la escasa eficacia del tratamiento.

Palabras-Ilave: Fibromialgia; Patología laboral; Valoración médico-legal.

\section{Introdución}

La fibromialgia (FM) se trata de una patología frecuente en nuestro país, con una prevalencia del 2,4\% de la población general mayor de 20 años, según los resultados obtenidos en el estudio Episer (RIVERA et al., 2006). En números absolutos, esto supone unos 700.000 pacientes afectados por la FM, en España. Por sexos, la prevalencia entre los varones se estima en un $0,2 \%$ frente a un $4,2 \%$ en las mujeres, lo que supone una relación mujer: varón de 21:1. Cuando afecta a la población infantil y juvenil, la mayor prevalencia en el sexo femenino no es tan acusada. La FM aparece en todos los grupos etarios, con una prevalencia máxima entre los 40 y 49 años, mientras que es relativamente infrecuente en personas que superan los 80 años de edad (RIVERA et al., 2006).

En los últimos años, la FM ha ido adquiriendo cada vez mayor importancia en nuestro medio hasta convertirse en un problema de salud pública de primer orden por su alta prevalencia en la población adulta. A esto se añade el insuficiente conocimiento de sus causas y mecanismos de producción ante la ausencia de tratamiento curativo, así como la creciente demanda médica y el consumo de recursos sanitarios que genera, el alto coste por falta de producción laboral y la repercusión social y laboral que padecen muchos de estos pacientes (RIVERA et al., 2006).

Por otra parte, no se ha establecido qué componentes de la FM están más relacionados con su gravedad y su repercusión laboral, ya que muchos de ellos son de apreciación subjetiva, y es difícil introducir instrumentos validados que permitan medir la discapacidad laboral en la FM. El propio proceso de evaluación de la discapacidad y los litigios por obtener o mejorar la compensación económica pueden actuar como factores agravantes del proceso. 
En Estados Unidos, entre el 6,3 y el 25,3\% de los pacientes con FM reciben pensiones de discapacidad, frente al 2,2\% de la población general y el 28,9\% de los pacientes con artritis reumatoide (WOLFE et al.,1997). En España, el estudio Episer sobre prevalencia de enfermedades reumáticas en la población española demostró como la FM tiene un notable impacto en la capacidad funcional, superado por el de la artritis reumatoide y superior al de la artrosis o la lumbalgia. Según este mismo estudio, el 11\% de las personas con fibromialgia se encuentra en incapacidad laboral temporal o permanente frente al 3,2\% de la población general. El $78 \%$ de los pacientes con FM que tiene trabajo remunerado ha estado en algún momento en situación de IT por la FM (EPISER, 2001).

No puede determinarse de forma genérica que la FM en sí misma conlleve a una incapacidad laboral, siendo preciso, por ello, el realizar una valoración individualizada dirigida fundamentalmente a los datos que permitan objetivar si la sintomatología referida por el paciente provoca una repercusión funcional de tal magnitud que impida el desarrollo de las tareas de su puesto de trabajo.

\section{Períodos medios de incapacidad temporal en trabajadores diagnosticados de fibromialgia}

En cuanto a los pacientes afectos de FM, se considera adecuado, caso de que precisen de una baja laboral, que la duración de la misma sea lo más corta posible, ya que se benefician claramente del mantenimiento de la actividad laboral, influyendo positivamente en su evolución. Es aconsejable que tan sólo en reagudizaciones severas y de forma autolimitada en el tiempo los pacientes causen baja por IT (SALIDO et al., 2007).

Las motivaciones que justifican reducir los períodos de baja laboral se fundamentan en que la situación de IT puede proporcionar resultados adversos, como ser más consciente del dolor que produce la inactividad, sentimiento de aislamiento, descenso de la actividad con la pérdida de la autoestima que esto conlleva, problemas económicos, pérdida de la estabilidad laboral, temor asociado al regreso al trabajo y problemas familiares.

En aquellos pacientes que precisen de una baja laboral dentro de un contexto médico-legal (tráfico, solicitud de minusvalía etc.), el facultativo ha de ser sumamente riguroso, ya que no es infrecuente que los beneficiarios pretendan prolongar el período de 
incapacidad. La IT estaría justificada para la realización de los estudios necesarios para descartar otras patologías.

El Manual de Tiempos Estándar publicado recientemente por el Instituto Nacional de Seguro Social (INSS) establece una duración promedio para la FM de 14 días (INSS, 2009). Una cuestión importante a tener en cuenta es el grado de insatisfacción laboral entre estos pacientes, el cual suele ser alto; considerando que sus condiciones laborales no son las adecuadas para su enfermedad, y además perciben un alto grado de rechazo y estrés laboral en su lugar de trabajo debido a su afección.

La estadística nos indica que asistimos a un aumento espectacular de casos en los últimos años y casi siempre en puestos de trabajo, calificados como de poco o nada gratificantes.

\section{Fibromialgia como patología generadora de incapacidad permanente}

Tanto para los órganos judiciales como para los administrativos, evaluar el grado de discapacidad de los pacientes con FM resulta una tarea compleja, y quizás derivada del planteamiento de una definición meramente sintomática, por lo cual no resulta fácil ni su valoración médica ni la determinación de su repercusión funcional (SSTJ, 2010). En este sentido, se comprueba que, la mera constatación del diagnóstico diferencial de FM no es elemento suficiente para establecer de forma automática el carácter incapacitante de esta enfermedad, sino que al tratarse de una patología cuyo elemento definitorio es el dolor generalizado de carácter esquelético y muscular, cuya intensidad no sólo puede variar de una a otra persona en función de los umbrales de sensibilidad al dolor de cada individuo, sino que incluso varía la intensidad en la misma persona en función de los días y de las horas del día (SSTJ, 2004), con lo cual la personalidad del paciente es un factor a tener en cuenta a la hora de efectuar valoraciones periciales. Por ello, es preciso puntualizar, que los informes clínicos que establezcan el diagnóstico de FM deberían estar emitidos por un médico especialista en reumatología, y que aquellos informes clínicos emitidos por el médico de familia y que resulten sin fundamentos científicos, y con finalidad de ser utilizados en la determinación de incapacidad permanente (IP) deben ser documentos descartados por falta de especialización de quien lo suscribe.

Frente a esta dificultad para determinar la existencia o no de IP de un afectado por FM, la jurisprudencia es muy taxativa en este sentido e indica que es preciso tener en 
cuenta tres parámetros que deberán ser relacionados y analizados de forma global para poder establecer la existencia o no de dicha incapacidad.

El primer parámetro sería las secuelas que presenta el afectado; en segundo lugar, la profesión que éste ejercita y, finalmente, el Reglamento de Accidentes de Trabajo, aprobado por el Decreto de 22 de junio 1956 (STSJ,1999 a; STSJ,2000 a; STSJ, 2000 b; STSJ, 2001). Es decir, los órganos judiciales combinan estos tres parámetros para determinar si el caso estudiado es susceptible de una IP.

En primer lugar, y respecto a las secuelas presentadas por el afectado, es necesario que las reducciones anatómicas o funcionales del mismo sean susceptibles de determinación objetiva. Es decir, que se puedan constatar médicamente de forma incuestionable, no basándose en la mera manifestación subjetiva del interesado (STSJ, 2003), ya que esta objetividad es una de las notas características que definen el concepto de IP (STSJ, 2006).

De hecho, el dolor derivado de la FM sin causa objetiva constatable ha venido siendo excluido para la calificación de las situaciones determinantes de IP. En esta línea, cuando no se constata el grado de FM junto con la sintomatología que ésta ocasiona al afectado ni el tratamiento que está recibiendo el trabajador, no se reconoce grado alguno de incapacidad. Además, las secuelas deben tener una previsión científica de carácter definitivo, es decir incurables e irreversibles y, con ello, la jurisprudencia establece que este tipo de previsión de irreversibilidad sería suficiente para poder determinar una IP (STSJ, 2006; STSJ, 2000; STSJ, 2001; STSJ, 2003; STSJ, 2003; STSJ, 2013).

En segundo lugar, y más allá de las secuelas que presente el paciente, la jurisprudencia (STSJ,1999 a; STSJ, 2000 a; STSJ, 2000 b; STSJ, 2001) también tiene en cuenta la profesión que el paciente desarrolla. En este sentido, para determinar la calificación del grado de IP no puede tenerse en cuenta la lesión en sí misma, sino que deberá analizarse la repercusión que ésta puede tener sobre la capacidad de trabajo del interesado. Además, deberá valorarse la capacidad laboral residual del afectado sin que puedan tomarse en consideración las circunstancias subjetivas de edad, preparación profesional y restantes de tipo económico y social que concurran, ya que estas causas subjetivas no pueden ser usadas para configurar un grado de incapacidad superior al que corresponde por razones objetivas de carácter médico (STSJ, 2013). 
Es decir, no basta con que las reducciones anatómicas y funcionales sean graves, sino que además es necesario que el sujeto se encuentre total o parcialmente incapacitado para trabajar, pues para reconocimiento de una IP sólo tienen relevancia aquellas lesiones que anulen o disminuyan la capacidad laboral (STSJ, 2006). Las lesiones existentes que no disminuyan o anulen la capacidad laboral del interesado, podrán ser susceptibles de una discapacidad (minusvalía), de acuerdo con el Real Decreto 1971/1999 de 23 de diciembre (STSJ, 2004), pero no causantes de una IP en cualquiera de sus grados.

De acuerdo con la jurisprudencia, no puede tenerse en cuenta la lesión en sí misma y debe analizarse detenidamente la repercusión que esta lesión pueda tener sobre el interesado. Y es que por muy grave que sea el cuadro clínico, si éste en conexión con los requerimientos que constituyen el núcleo de la actividad profesional no alcanza relevancia para el desarrollo efectivo de un trabajo, ello impide la calificación como IP. Por el contrario, una dolencia o lesión aparentemente insignificante puede repercutir anulando la concreta realización de una profesión (STSJ, 2006). En otras palabras, pérdidas pequeñas de la capacidad global pueden justificar una IP total y pérdidas grandes pueden no ser suficientes para ello si la profesión es sedentaria o de gran variedad funcional (STSJ, 2003; STSJ, 2013; STSJ, 2004). De manera que, el cuadro patológico que presenta el interesado se debe analizar en relación con el trabajo o profesión que éste desarrolla, ya que, según la profesión habitual del afectado la lesión impedirá o no la realización de las tareas fundamentales de la misma.

Finalmente y en tercer lugar, los órganos judiciales (STSJ, 1999 a; STSJ, 2000 a; STSJ, 2000 b; STSJ, 2001) ponderan, para determinar la existencia o no de IP, como criterio orientativo, el artículo 38 o el 41 (según el grado de incapacidad solicitado) del Decreto 22 de junio de 1956. Y es que, además de la profesión habitual y de las secuelas, y como consecuencia de la ausencia de listas y baremos de uso común para calificar la FM, los Tribunales han recuperado el baremo previsto en el Reglamento de Accidentes de Trabajo para poder calificar el grado de IP a partir de las patologías sufridas.

Por todo lo anteriormente expuesto, no todos los pacientes con FM serán beneficiarios de una IP. De hecho, la inmensa mayoría de los afectados por esta patología pueden mantener, con adaptaciones, su actividad laboral (entre el 53 y el 70 por ciento del total de afectados) (STSJ, 2006). 
En sentido opuesto, se calcula que, de todos los afectados por la enfermedad, entre el 20 y el 30 por ciento de los mismos requerirá una incapacidad para su profesión habitual aunque ésta sufra adaptaciones. Es decir, entre un 20 y un 30 por ciento de los pacientes necesitan una IP total. Además, se establece que entre un 10 y un 17 por ciento de los enfermos de FM requieren una IP absoluta, que es aquella incapacidad que inhabilita para todo trabajo al sujeto afectado. Cuando se reconoce una IP total es porque el Tribunal considera que el interesado presenta secuelas de carácter definitivo, permanente, progresivo e irreversible (STSJ, 2002) que no le permiten realizar trabajos (normalmente) de esfuerzo físico y/o psíquico, trabajos que requieran deambulación prolongada o trabajos manuales (STSJ, 1999 b), pues la realización de las tareas fundamentales de estas profesiones comportaría un padecimiento no exigible al trabajador (STSJ, 2003). Sin embargo, el cuadro patológico presentado por estos enfermos si les permite realizar actividades livianas o sedentarias, pues mantienen capacidad residual suficiente para desarrollar una profesión diferente de la que se ha usado para declarar dicha incapacidad (STSJ, 1997; STSJ, 2001; STSJ, 2003; STSJ, 2004; STSJ, 2009 b; STSJ, 2010 b). De manera que, según los Tribunales, estos pacientes no están incapacitados para realizar una actividad que requiera un horario establecido e instrucciones empresariales, así como eficacia y rendimiento mínimos (STSJ, 2001).

\section{Discusión}

Como resumen de lo plasmado anteriormente y recogido en el presente artículo, afirmamos que la FM es una enfermedad generadora de situaciones de IT y susceptible de ser apreciada como una contingencia responsable de IP, tanto total como absoluta, ya que altera la vida cotidiana de las personas que la padecen y tiene consecuencias para el desarrollo normal de la actividad laboral del trabajador afectado.

En situaciones de IT, el trabajador es beneficiario de unas prestaciones sanitarias y económicas por la pérdida de ingresos que provoca la incapacidad para trabajar. En muchas ocasiones, la baja laboral por IT, puede proporcionar a este tipo de pacientes resultados adversos ya que, al no tener una ocupación, son más conscientes de las repercusiones que conlleva esta enfermedad. 
Sólo en aquellos casos más graves, la FM puede ser generadora de una IP, y cada situación deberá ser estudiada de forma individual pues los afectados no tienen el mismo cuadro patológico y, aunque así fuera, este cuadro no afecta de idéntica forma a todos los pacientes. Además, estos pacientes tampoco desarrollan la misma actividad laboral, teniendo cada interesado, unas actividades fundamentales propias de su profesión habitual. De manera que la combinación de las patologías con el tipo de actividad laboral que desarrolle el trabajador así como la repercusión de las primeras sobre el desarrollo de las tareas profesionales del afectado determinará la existencia o no de IP en alguno de sus grados.

Al tratarse de una patología con distinta repercusión en la vida cotidiana y la vida laboral, tanto para los órganos administrativos como para los judiciales resulta sumamente complejo evaluar el grado de discapacidad de los enfermos de FM.

A nivel genérico, la jurisprudencia reconoce una IP absoluta derivada de la patología estudiada cuando las limitaciones funcionales presentadas por el trabajador son altamente incapacitantes y, por lo tanto, le impiden dedicarse a cualquier actividad laboral por liviana que sea (STSJ, 2010 a), con la habitualidad y el rendimiento normalmente exigible (STSJ, 2000). De modo que la situación patológica es incompatible con cualquier trabajo que exija o no esfuerzo (STSJ, 2008) pues, aunque la actividad laboral no requiera esfuerzo alguno, los desplazamientos diarios al trabajo y la sujeción a una jornada laboral si lo exigen (STSJ, 2009 a).

En esta dirección, un paciente con una sintomatología de este tipo debe declararse en IP absoluta, pues es la única conclusión jurídica, humana y de justicia existente al no conservar ningún tipo de capacidad laboral residual.

En sentido contrario y en líneas generales, los Tribunales no otorgan una IP por FM cuando el cuadro patológico no se acredita mediante un informe realizado por un médico especialista (reumatólogo) o existen informes médicos contradictorios o no coincidentes (STSJ, $2000 \mathrm{c}$ ).

\section{Referencias bibliograficas}

EPISER. Prevalencia e impacto de las enfermedades reumáticas en la población adulta española (EPISER 2000). Madrid: Sociedad Española de Reumatología, 2001. 
ESPAÑA. ANDALUCÍA. Tribunal Superior de Justicia (STSJ). Sentencia $n$. JUR|2003|74680. Andalucía, 19 de diciembre 2003.

ESPAÑA. ASTURIAS. Tribunal Superior de Justicia (STSJ). Sentencia n. JUR|2013|88919. Asturias, 1 de febrero 2013.

ESPAÑA. ASTURIAS. Tribunal Superior de Justicia (STSJ). Sentencia n. JUR|2001|145. Asturias, 17 de noviembre 2000 .

ESPAÑA. CANTABRIA. Tribunal Superior de Justicia (STSJ). Sentencia $n$. JUR|2010|357614. Cantabria, 21 de abril 2010.

ESPAÑA. CANTABRIA. Tribunal Superior de Justicia (STSJ). Sentencia n. AS|2001|3939. Cantabria, 19 de septiembre 2001.

ESPAÑA. CASTILLA Y LEÓN. Tribunal Superior de Justicia (STSJ). Sentencia $n$. JUR|2013|115291. Castilla y León, 20 de marzo 2013.

ESPAÑA. CASTILLA Y LEÓN. Tribunal Superior de Justicia (STSJ). Sentencia $n$. JUR|2004|254939. Castilla y León, 13 de septiembre 2004.

ESPAÑA. CASTILLA Y LEÓN. Tribunal Superior de Justicia (STSJ). Sentencia $n$. JUR|2003|84614. Castilla y León, 10 de febrero 2003.

ESPAÑA. CASTILLA-LA MANCHA. Tribunal Superior de Justicia (STSJ). Sentencia $n$. AS|2002|196. Castilla-La Mancha, 28 de diciembre 2001.

ESPAÑA. CATALUÑA. Tribunal Superior de Justicia (STSJ). Sentencia $n$. JUR|2010|382893. Cataluña, 22 de octubre 2010. (a)

ESPAÑA. CATALUÑA. Tribunal Superior de Justicia (STSJ). Sentencia n. JUR|2010|374540. Cataluña, 28 de septiembre 2010. (b)

ESPAÑA. CATALUÑA. Tribunal Superior de Justicia (STSJ). Sentencia $n$. JUR|2004|314518. Cataluña, 1 de octubre 2004.

ESPAÑA. CATALUÑA. Tribunal Superior de Justicia (STSJ). Sentencia n. AS1200015434. Cataluña, 31 de enero 2000.

ESPAÑA. EXTREMADURA. Tribunal Superior de Justicia (STSJ). Sentencia $n$. AS|2003|3866. Extremadura, 8 de octubre 2003.

ESPAÑA. Instituto Nacional de la Seguridad Social (INSS). Manual de tiempos estándar de Incapacidad Temporal. Madrid: INSS, 2009.

ESPAÑA. MADRID. Tribunal Superior de Justicia (STSJ). Sentencia n. JUR|2006|154878. Madrid, 27 de febrero 2006.

ESPAÑA. MADRID. Tribunal Superior de Justicia (STSJ). Sentencia n. AS|2004|3916. Madrid, 29 de noviembre 2004.

ESPAÑA. MADRID. Tribunal Superior de Justicia (STSJ). Sentencia n. AS1200313889. Madrid, 27 de julio 2003.

ESPAÑA. MADRID. Tribunal Superior de Justicia (STSJ). Sentencia n. ASI2002|3313. Madrid, 17 de septiembre 2002. 
ESPAÑA. MURCIA. Tribunal Superior de Justicia (STSJ). Sentencia n. JUR|2009|496927. Murcia, 2 de noviembre 2009. (a)

ESPAÑA. MURCIA. Tribunal Superior de Justicia (STSJ). Sentencia n. JUR|2009|376096. Murcia, 30 de junio 2009. (b)

ESPAÑA. MURCIA. Tribunal Superior de Justicia (STSJ). Sentencia n. JUR|2008|359815. Murcia, 14 de octubre 2008.

ESPAÑA. NAVARRA. Tribunal Superior de Justicia (STSJ). Sentencia n. AS|2003|4072. Navarra, 30 de octubre 2003.

ESPAÑA. MURCIA. Tribunal Superior de Justicia (STSJ). Sentencia n. JURI2001|331957. Murcia, 22 de octubre 2001.

ESPAÑA. MURCIA. Tribunal Superior de Justicia (STSJ). Sentencia n. JURl2000165625. Murcia, 17 de febrero 2000. (a)

ESPAÑA. MURCIA. Tribunal Superior de Justicia (STSJ). Sentencia n. JURl20001257424. Murcia, 3 de julio 2000. (b)

ESPAÑA. MURCIA. Tribunal Superior de Justicia (STSJ). Sentencia n. JUR|2000165626. Murcia, 17 de enero 2000. (c)

ESPAÑA. MURCIA. Tribunal Superior de Justicia (STSJ). Sentencia n. JUR|1999|240550. Murcia, 6 de septiembre 1999. (a)

ESPAÑA. MURCIA. Tribunal Superior de Justicia (STSJ). Sentencia n. JUR|1999|128514. Murcia, 2 de febrero 1999. (b)

ESPAÑA. MURCIA. Tribunal Superior de Justicia (STSJ). Sentencia n. JUR|1999|6775. Murcia, 24 de noviembre 1997.

ESPAÑA. NAVARRA. Tribunal Superior de Justicia (STSJ). Sentencia n. JUR|2012|97391. Navarra, 28 de noviembre 2011.

LAFFON, A. y ALONSO A. Manual S.E.R. de las enfermedades reumáticas. Madrid: Médica Panamericana. 2001.

RIVERA, J. et al. Documento de consenso de la Sociedad Española de Reumatología sobre la fibromialgia. Reumatología Clínica, v.2, p. S55-S66, 2006.

RIVERA, J. et al. Evidencias terapéuticas en fibromialgia. Reumatología Clínica, 2:S34S37, 2006.

SALIDO, M. et al. Factores relacionados con la incapacidad temporal en pacientes con fibromialgia. Reumatología Clínica, v.3, p.67-72, 2007.

WOLFE, F. et al. Work and disability status of person with fibromyalgia. Journal of Rheumatology, v.24, p.1171-8, 1997. 